\title{
Diastolic Paradoxic Jet Flow of the Right Ventricle in Hypertrophic Cardiomyopathy
}

\author{
Ayumi Shirota ${ }^{\mathrm{a}, \mathrm{e}}$, Tatsuya Kawasaki ${ }^{\mathrm{a}}$, Michiyo Yamano ${ }^{\mathrm{a}, \mathrm{b}}$, Takatomo Shima ${ }^{\mathrm{c}}$, \\ Takashi Nakamura ${ }^{\mathrm{d}}$, Satoaki Matoba ${ }^{\mathrm{b}}$
}

\begin{abstract}
An early diastolic flow from the left ventricular apex to the base can be shown in patients with hypertrophic cardiomyopathy (HCM). This tiny flow or a diastolic paradoxic jet flow is important to detect on echocardiography because of its association with cardiovascular adverse events. We report an asymptomatic 44-year-old man with midventricular obstructive HCM, in which a diastolic paradoxic jet flow was observed not only in the left ventricle but also in the right ventricle. The diastolic paradoxic jet flow in the right ventricle started approximately $110 \mathrm{~ms}$ after the onset of the second heart sound, lasted for almost $95 \mathrm{~ms}$, and disappeared in coincidence with the third heart sound; the onset was later and the duration was similar, compared with the diastolic paradoxic jet flow in the left ventricle. He had been doing well without any medication for months and later lost to follow-up.
\end{abstract}

Keywords: Hypertrophic cardiomyopathy; Paradoxic jet flow; Right ventricle

\section{Introduction}

Patients with hypertrophic cardiomyopathy (HCM) can have an early diastolic flow from the apex to the base of the left ventricle (a diastolic paradoxic jet flow) with an estimated incidence of $10 \%$ [1-3]. This tiny flow should be carefully detected on echocardiographic examination, since the presence of a diastolic paradoxic jet flow in the left ventricle suggests an increased risk of adverse cardiovascular events [3-5]. We report a case of HCM with a paradoxic jet flow not only in the

Manuscript submitted October 31, 2018, accepted November 12, 2018

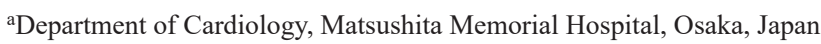
bDepartment of Cardiovascular Medicine, Graduate School of Medical Science, Kyoto Prefectural University of Medicine, Kyoto, Japan

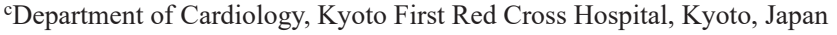

${ }^{\mathrm{d}}$ Department of Cardiology, Saiseikai Shiga Hospital, Shiga, Japan

${ }^{e}$ Corresponding Author: Ayumi Shirota, Department of Cardiology, Matsushita Memorial Hospital, Sotojima 5-55, Moriguchi, Osaka 570-8540, Japan.

Email: ashirota@koto.kpu-m.ac.jp

doi: https://doi.org/10.14740/jmc3210 left ventricle but also in the right ventricle.

\section{Case Report}

An asymptomatic 44-year-old man presented to Matsushita Memorial Hospital to seek medical follow-up after moving. The patient was found to have an abnormal electrocardiogram (i.e. left ventricular hypertrophy) at a medical checkup several years ago and was diagnosed with HCM at another hospital. He reported to have received bisoprolol $5 \mathrm{mg}$ per day, which was discontinued for his preference. His previous medical history was otherwise unremarkable. Cardiac auscultation at the apex showed a fourth sound (S4), a grade 3 systolic ejection murmur, and a third heart sound (S3). The remainder of the examination was normal. There was no family history of HCM or premature sudden death.

Echocardiography showed myocardial hypertrophy in the interventricular septum, anterior and lateral walls, and the apex of the left ventricle (Fig. 1a-c). The left ventricular ejection fraction was $71 \%$, the left atrium dimension was 38 $\mathrm{mm}$, the peak transmitral $\mathrm{E}$ wave velocity and the deceleration time of the transmitral $\mathrm{E}$ wave were $0.73 \mathrm{~m} / \mathrm{s}$ and 153 $\mathrm{ms}$, and the transmitral E/A ratio and E/early diastolic mitral annular velocity ratio were 2.35 and 20.85 , respectively. Midventricular obstruction with a peak velocity of $3.2 \mathrm{~m} / \mathrm{s}$ was observed in the left ventricle, along with a diastolic paradoxic jet flow (Fig. 1d). Of note, right ventricular hypertrophy was also suspected, and a tiny flow from the right ventricular apex to the base during early diastole was detected (Fig. 1e). The unique flow started approximately $110 \mathrm{~ms}$ after the onset of the second heart sound (S2) and lasted to the end of the S3 or for almost $95 \mathrm{~ms}$ (Fig. 2). The onset was likely to be later than the diastolic paradoxic jet flow in the left ventricle, and the duration seemed similar to that of the left ventricle, although the tiny flow was difficult to assess by pulsed Doppler echocardiography.

Cardiac magnetic resonance revealed myocardial hypertrophy in the ventricular septum, the right ventricular free wall, and left ventricular anterior and lateral walls (Fig. 3). Prominent trabeculation was not present in the left ventricle as well as in the right ventricle. Extensive late gadolinium enhancement was detected mainly in the areas of myocardial hypertrophy. Medical treatment including beta-blockers or anticoagulants was recommended but declined by the patient. He had 

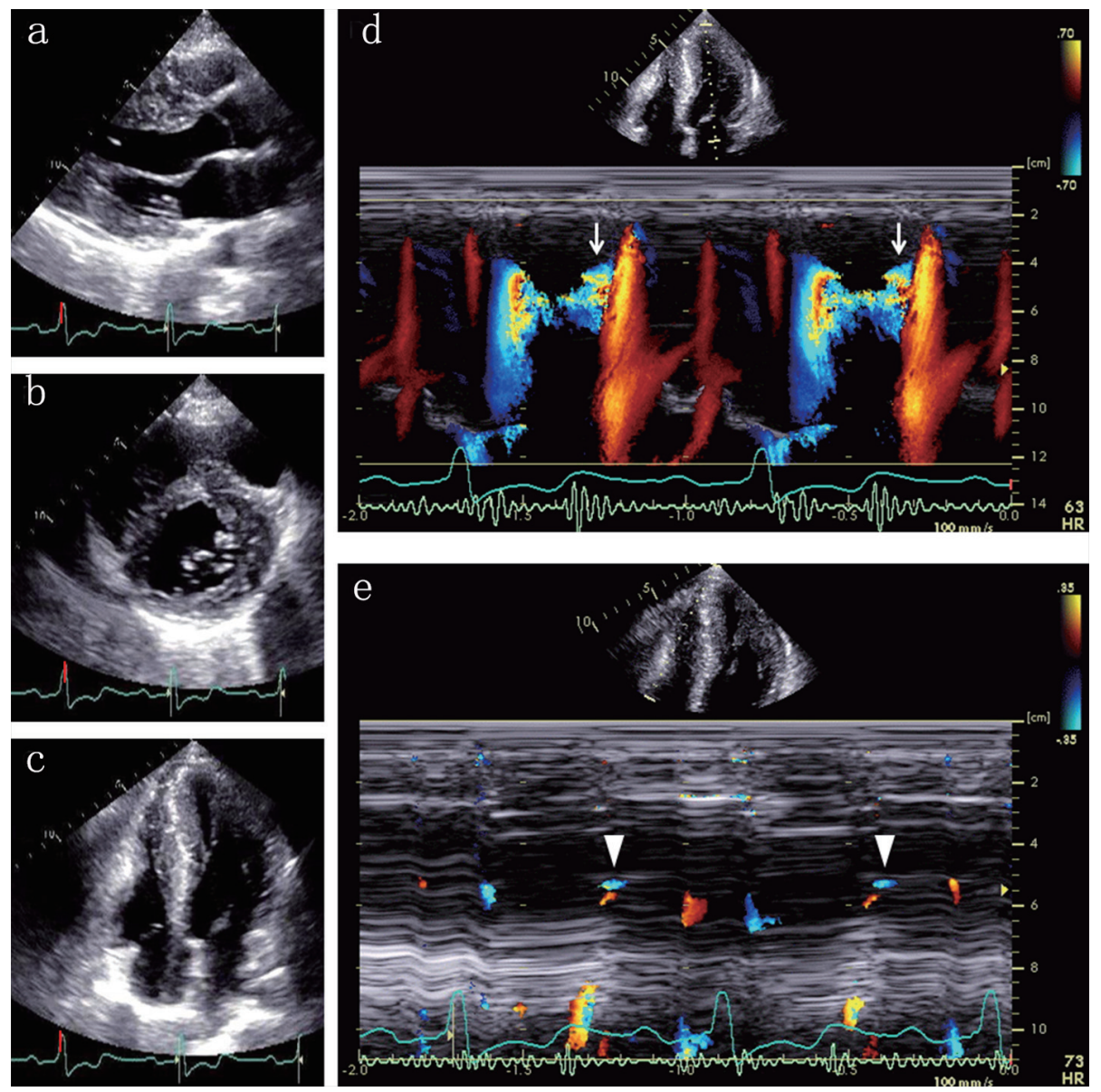

Figure 1. Echocardiographic images at end-diastole show myocardial hypertrophy in the ventricular septum, left ventricular anterior and lateral walls, and the apex of both ventricles ((a) parasternal long axis view; (b) parasternal short axis view; (c) apical four-chamber view). Color Doppler M-mode shows a diastolic paradoxic jet flow from the apex toward the base of the left ventricle ((d) arrows). Note a similar flow in the right ventricle ((e) arrowheads).

been doing well without any medication for months and later lost to follow-up.

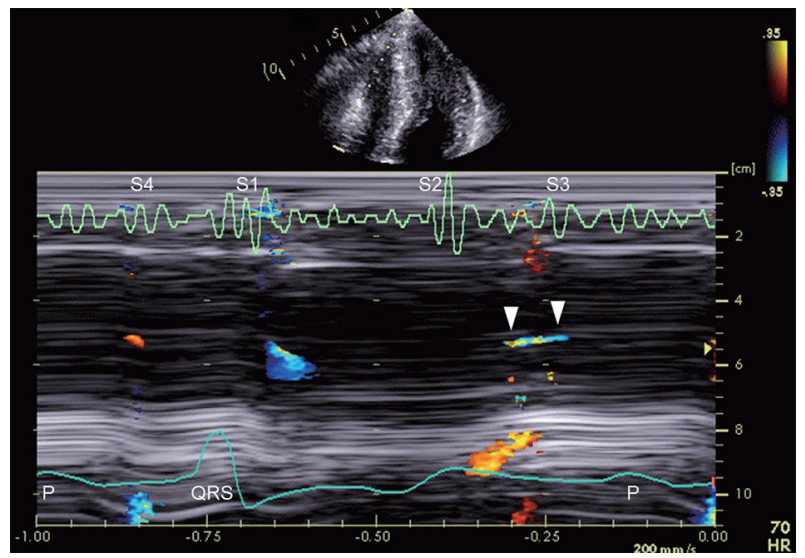

Figure 2. Color Doppler M-mode shows an early diastolic flow from the apex toward the base of the right ventricle (arrowheads). The unique flow lasts to the third heart sound (S3). S1 indicates first heart sound; $\mathrm{S} 2$, second heart sound; and S4, fourth heart sound.

\section{Discussion}

The current case showed an early diastolic flow from the apex to the base in the right ventricle. This uncommon phenomenon may be observed in the setting of prominent trabeculation (e.g. non-compaction cardiomyopathy or end-stage renal disease), but the possibility is less likely in the current case, since no prominent trabeculation was detected on cardiac magnetic resonance. We may safely consider that the unique flow in the right ventricle was a diastolic paradoxic jet flow related to right ventricular hypertrophy, given its similarity to the diastolic paradoxic jet flow in the left ventricle.

Proposed mechanisms of a diastolic paradoxic jet flow in the left ventricle are an increased pressure gradient between the high-pressure apical chamber and the low-pressure basal part of the left ventricle during early diastole [3]. It is reasonable to consider that a diastolic paradoxic jet flow in the right ventricle could be provoked by the same conditions of a diastolic paradoxic jet flow in the left ventricle; however, in our case, a right ventricular apical pouch was not evident on echocardiography and cardiac magnetic resonance, the latter of which is the most 

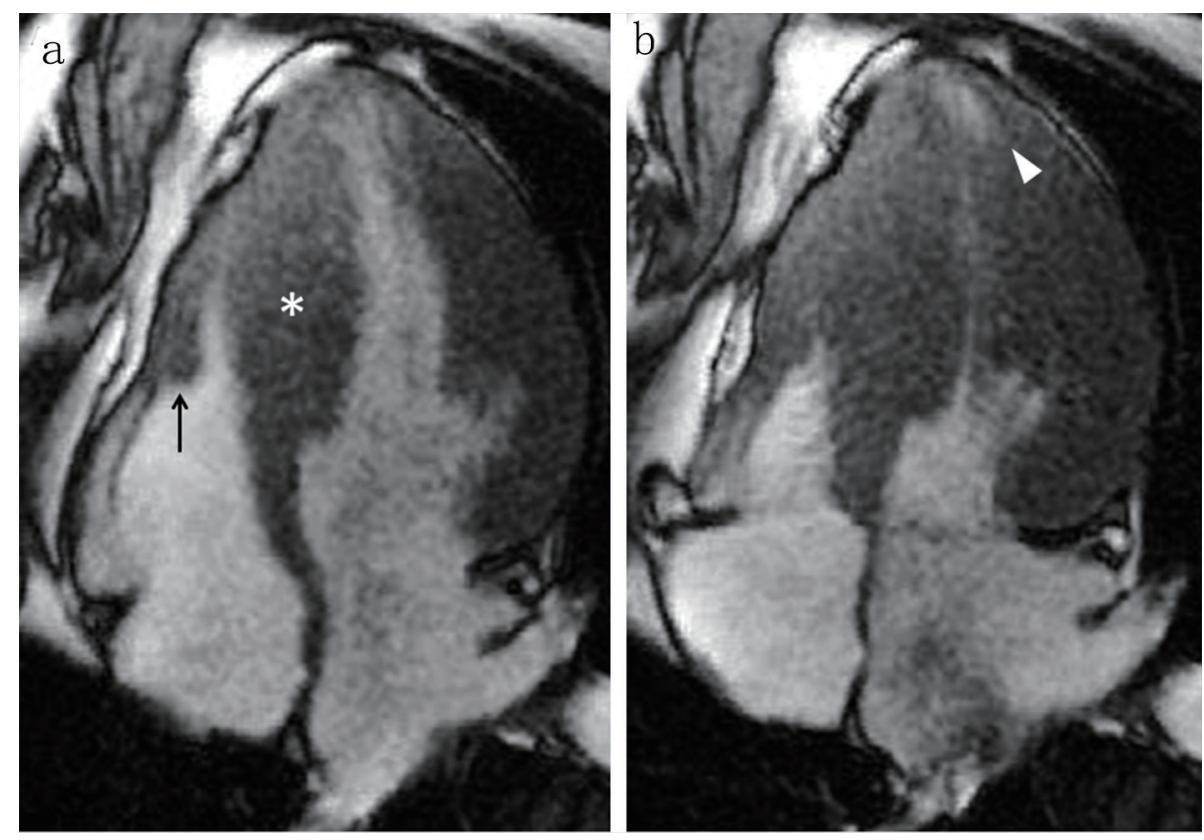

Figure 3. A four-chamber cine cardiac magnetic resonance image at end-diastole shows myocardial hypertrophy in the ventricular septum ((a) asterisk), the right ventricular free wall ((a) arrow), and the left ventricular lateral wall. An apical pouch is present in the left ventricle at end-systole ((b) arrowhead), but not evident in the right ventricle.

recommended for the identification of a left ventricular apical pouch [6]. The onset of the diastolic paradoxic jet flow in the right ventricle was later than that of the left ventricle. The difference may be explained by the fact that the aortic component of S2 usually occurs prior to the pulmonic component [7,8], which means that the left ventricle precedes the right ventricle regarding the onset of diastole. The possible effects of myocardial hypertrophy on interventricular conduction delay or interventricular septum bulging due to mid-ventricular obstruction on the right ventricle should also be considered.

Conditions associated with a diastolic paradoxic jet flow in the left ventricle include the coexistence of left ventricular hypertrophy and apical obstruction. In a large cohort of 2,650 patients with $\mathrm{HCM}$, the prevalence of right ventricular hypertrophy defined as a maximum right ventricular wall thickness $\geq 10 \mathrm{~mm}$ was $1.3 \%$ [9]. It is also reported in another study [10] that apical obstruction of the right ventricle, defined as a high flow velocity signal of more than $2.0 \mathrm{~m} / \mathrm{s}$ on continuous wave Doppler echocardiography, was observed in approximately $3 \%$ of HCM patients. Taken together, it seems quite rare that a diastolic paradoxic jet flow in the right ventricle is observed among HCM patients, because right ventricular hypertrophy and obstruction are likely to be required for the uncommon flow. To our knowledge, our patient is the first case in which a diastolic paradoxic jet flow was clearly demonstrated in the right ventricle, although this phenomenon was suggested by detailed analysis of continuous wave Doppler data in relation to the onset of the tricuspid rapid filling wave in HCM patients with obstruction in the right ventricular apex [10].

The presence of a diastolic paradoxic jet flow in the left ventricle has been significantly associated with higher risks of systemic embolism and ventricular tachycardia $[3,5]$. Further- more, a diastolic paradoxic jet flow has been also significantly associated with thallium-201 myocardial perfusion abnormalities localized to the left ventricular apical region [3], with the severity increasing according to the duration of apical cavity obliteration [4]. In our case, the duration of apical cavity obliteration was unmeasurable in the right ventricle because of its inconspicuousness. It remains unclear whether a diastolic paradoxic jet flow in the right ventricle, regardless of the presence of a diastolic paradoxic jet flow in the left ventricle, would be related to adverse outcomes. Further research is warranted to examine the clinical characteristics of a diastolic paradoxic jet flow in the right ventricle or in both ventricles.

\section{Acknowledgments}

None to declare.

\section{Financial Disclosure}

None to declare.

\section{Conflict of Interest}

None to declare.

\section{Informed Consent}

Not applicable. 


\section{Author Contributions}

AS, TK, and MY contributed to data analysis and study design; AS and TK wrote the paper; TS, TN, and SM reviewed the paper; all authors gave the final approval.

\section{Data Availability}

The authors declare that data supporting the findings of this study are available within the article.

\section{References}

1. Shiota T, Sakamoto T, Takenaka K, Suzuki J, Amano W, Igarashi T, Amano K, et al. [Paradoxical left ventricular blood flow during the isovolumic relaxation period in non-obstructive hypertrophic cardiomyopathy: Doppler and M-mode echocardiographic study]. J Cardiol. 1990;20(1):83-93.

2. Nakamura T, Furukawa K, Matsubara K, Kitamura H, Sugihara H, Inoue D, Asayama J, et al. [Long-term followup of electrocardiographic changes in patients with asymmetric apical hypertrophy]. J Cardiol. 1990;20(3):635647.

3. Nakamura T, Matsubara K, Furukawa K, Azuma A, Sugihara H, Katsume H, Nakagawa M. Diastolic paradoxic jet flow in patients with hypertrophic cardiomyopathy: evidence of concealed apical asynergy with cavity oblitera- tion. J Am Coll Cardiol. 1992;19(3):516-524.

4. Matsubara K, Nakamura T, Kuribayashi T, Azuma A, Nakagawa M. Sustained cavity obliteration and apical aneurysm formation in apical hypertrophic cardiomyopathy. J Am Coll Cardiol. 2003;42(2):288-295.

5. Noguchi A, Ishizuka N, Tanimoto K, Kasanuki H. [Follow-up study of diastolic paradoxical flow in the left ventricle with apical hypertrophic cardiomyopathy]. J Cardiol. 2006;47(1):15-23.

6. Maron MS, Finley JJ, Bos JM, Hauser TH, Manning WJ, Haas TS, Lesser JR, et al. Prevalence, clinical significance, and natural history of left ventricular apical aneurysms in hypertrophic cardiomyopathy. Circulation. 2008;118(15):1541-1549.

7. Curtiss EI, Matthews RG, Shaver JA. Mechanism of normal splitting of the second heart sound. Circulation. 1975;51(1):157-164.

8. Felner JM. The second heart sound. In: Walker HK, Hall WD, Hurst JW, editors. Clinical methods: the history, physical, and laboratory examinations. 3rd ed. Boston: Butterworths; 1990. p. 122-125.

9. Guo X, Fan C, Tian L, Liu Y, Wang H, Zhao S, Duan $\mathrm{F}$, et al. The clinical features, outcomes and genetic characteristics of hypertrophic cardiomyopathy patients with severe right ventricular hypertrophy. PLoS One. 2017;12(3):e0174118.

10. Shimizu M, Kawai H, Yokota Y, Yokoyama M. Echocardiographic assessment of right ventricular obstruction in hypertrophic cardiomyopathy. Circ J. 2003;67(10):855860. 